Original paper

\title{
Transient elastography reliably estimates liver fibrosis in autoimmune hepatitis
}

\author{
Nadim Mahmud ${ }^{1,2}$, Sahil D. Doshi ${ }^{1}$, Kimberly A. Forde ${ }^{1,3}$, Vandana Khungar ${ }^{1}$ \\ 'Division of Gastroenterology, University of Pennsylvania Perelman School of Medicine, Philadelphia PA, United States \\ ¿Leonard Davis Institute of Health Economics, University of Pennsylvania, Philadelphia PA, United States \\ ${ }^{3}$ Center for Clinical Epidemiology and Biostatistics, University of Pennsylvania Perelman School of Medicine, Philadelphia PA, United States
}

\begin{abstract}
Aim of the study: Autoimmune hepatitis (AIH) may result in liver fibrosis and cirrhosis. While the gold standard for staging fibrosis is biopsy, transient elastography (TE) represents a non-invasive alternative. TE has been validated in several chronic liver diseases, but no data exist to establish an association between histologic fibrosis on biopsy and TE liver stiffness measurements among a United States cohort of AlH patients.

Material and methods: We conducted a retrospective cohort study of 53 AlH patients who received TE assessment and liver biopsy. Histologic fibrosis was classified as advanced (F3-F4) or mild/moderate (FO-F2). Liver stiffness by TE was measured in kilopascals (KPa). We performed a score test for trend to test the association between histologic fibrosis stage and increasing TE kPa categories. Analyses incorporated probe type (medium or extra-large) and body mass index (BMI). Linear regression was used to generate predicted associations between median $\mathrm{kPa}$ and histologic fibrosis score with the medium probe.

Results: The cohort was primarily female (83\%) with median age 56.3 years. Increasing kPa category was associated with worsening fibrosis stage when using the medium probe $(p=0.04)$, but not the extra-large probe

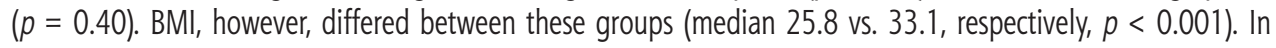
adjusted linear regression, increasing median $\mathrm{kPa}$ corresponded well to worsening fibrosis stage $(p=0.003)$.

Conclusions: In a United States AlH cohort, increasing TE kPa measurements are associated with worsening histologic fibrosis staging. While medium probe performance was superior to the extra-large probe, significant variation in BMI between groups may explain this difference.
\end{abstract}

Key words: transient elastography, autoimmune hepatitis, FibroScan, liver biopsy, fibrosis staging.

Address for correspondence

Nadim Mahmud, MD, MS, MPH, Division of Gastroenterology, University of Pennsylvania Perelman School of Medicine, 3400 Civic Center Boulevard, $4^{\text {th }}$ Floor, South Pavilion, Philadelphia, PA 19104, United States, phone: 215-349-8222, fax: 215-349-5915, e-mail: nadim.mahmud@uphs.upenn.edu

\section{Introduction}

Autoimmune hepatitis (AIH) is a condition whereby chronic inflammation in the liver may result in progressive fibrosis and eventually cirrhosis. AIH is diagnosed through a combination of biochemical and serological markers, including increased levels of serum aminotransferases, high immunoglobulins, and the presence of circulating autoantibodies $[1,2]$. Liver biopsy plays an important role in the diagnosis and follow-up of patients with AIH by allowing for disease staging and assessment of inflammation and fibro- sis. While the gold standard for staging liver fibrosis is liver biopsy [3], this is an invasive procedure with an inherent risk of complications. It is also subject to sampling error and/or variations in technique that may result in incorrect staging of disease $[4,5]$. Several technologies, such as transient elastography (TE), have therefore emerged as non-invasive means of estimating liver fibrosis.

TE is commonly used across the world and was approved for use in the United States by the Food and Drug Administration in April 2013 [4, 6]. The association between TE measurements and liver fibrosis has 
been previously validated in several other chronic liver diseases, including alcoholic liver disease, non-alcoholic fatty liver disease, hepatitis $B$, and hepatitis $C$ [4, 7-10]. However, data regarding the validity of TE in $\mathrm{AIH}$ patients remain limited, with existing data coming exclusively from Asian or German cohorts [11-15]. To date no studies have focused on a North American cohort, nor have they studied the impact of body mass index (BMI) on TE measurements for AIH patients. Thus, In this study, we aimed to determine the association between histologic liver fibrosis and TE measurements of liver stiffness in demographics reflective of the United States AIH population. The impact of probe type and BMI on this relationship was also evaluated.

\section{Material and methods}

\section{Design and cohort creation}

This was a retrospective cohort study performed at the Hospital of the University of Pennsylvania in order to determine the association between TE assessment of liver stiffness, as measured in kilopascals $(\mathrm{kPa})$, and histologic fibrosis staging. We included patients age 18-85 who underwent TE for an indication of AIH between December 2014 and May 2017, and who received liver biopsy, through either the percutaneous or the transjugular approach. Diagnoses of AIH were confirmed through manual chart review of clinical documentation and laboratory data. In general, at our institution $\mathrm{AIH}$ is diagnosed according to American Association for the Study of Liver Disease guidelines [16]. In brief, patients are diagnosed with AIH if there is at least one elevation of an aminotransferase, typically at least 2 times the upper limit of the normal range, as well as elevation of an associated autoantibody (e.g., anti-smooth muscle antibody, antinuclear antibody, anti-liver/kidney microsomal-1 antibody). In patients suspected of having AIH with negative antibodies, liver biopsy is pursued to evaluate for histologic evidence of AIH. At our institution, in patients with established $\mathrm{AIH}$, liver biopsy is also commonly performed prior to tapering therapy, as was done for many patients in our cohort. In this study, we excluded patients who had not received a liver biopsy. We also excluded patients who had concomitant liver disease in addition to $\mathrm{AIH}$, or who had unreliable TE measurements (described below). Finally, this study met criteria for University of Pennsylvania Institutional Review Board exemption.

\section{Variable collection}

Electronic medical records for included patients were reviewed in order to obtain demographic data (age, sex, race). BMI, laboratory data (sodium, creatinine, albumin, total bilirubin, alkaline phosphatase, aspartate aminotransferase [AST], alanine aminotransferase [ALT], international normalized ratio, and platelet count), model for end-stage liver disease (MELD) computation, and comorbidities (hypertension, hyperlipidemia, diabetes, other concomitant liver disease) were obtained at the time of TE measurement. We also obtained AIH treatment data including active therapy with steroids, azathioprine, tacrolimus, or mycophenolate mofetil, and created a binary variable to indicate any active therapy versus no therapy. All liver biopsy pathology reports were manually reviewed, and degree of liver fibrosis was graded using the Batts-Ludwig scale (F0 to F4, where F0 - no fibrosis and F4 severe fibrosis). For pooled analyses, subcategories of advanced fibrosis (F3-F4) and mild fibrosis (F0-F2) were also created. In all included cases, liver biopsy specimens were adequate for fibrosis staging.

\section{Transient elastography measurement}

TE measurements of liver stiffness were acquired using a FibroScan device (Echosens, Paris, France). Patients were positioned in the supine position with their right arm maximally abducted. The probe tip was positioned over an intercostal space in order to target the right lobe of the liver. The medium $(\mathrm{M})$ probe (3.5 MHz) or extra-large (XL) probe $(2.5 \mathrm{MHz})$ was selected per operator discretion. Using A-mode ultrasound image feedback from the FibroScan device, a region of liver parenchyma with at least $6 \mathrm{~cm}$ thickness was identified, and ten $\mathrm{kPa}$ stiffness measurements were subsequently acquired. Readings were regarded to be reliable if the interquartile range (IQR) to median liver stiffness ratio (IQR/M) was $\leq 30 \%$, consistent with the Society of Radiologists in Ultrasound consensus and the most recent recommendations from the literature [17-20]. Of those with reliable results, the median $\mathrm{kPa}$ was used as the liver stiffness measure.

\section{Statistical analysis}

Descriptive statistics were calculated for patient characteristics, with medians and interquartile range (IQR) presented for continuous variables. Bar graphs for median $\mathrm{TE} \mathrm{kPa}$ for each fibrosis category were created, stratified by probe type, in order to visualize the distribution of TE measurements relative to histologic fibrosis. These plots were used to guide creation of five $\mathrm{kPa}$ categories for further analysis $(\leq 5.2,5.3-6.1$, $6.2-8.4,8.5-14.6, \geq 14.7$ ), based on approximate correspondence to fibrosis stage. Score tests for trend were 
Table 1. Patient characteristics

\begin{tabular}{lc}
\hline Variable & Value $(N=53)$ \\
\hline Age, median (IQR) & $56.3(44.4,63.5)$ \\
\hline Female sex & $44(83 \%)$ \\
\hline BMI, median (IQR) & $27.7(24.02,31.65)$ \\
\hline Race & $36(68 \%)$ \\
\hline White & $11(21 \%)$ \\
\hline Black & $6(11 \%)$ \\
\hline Other & $7(13 \%)$ \\
\hline Diabetes & $20(38 \%)$ \\
\hline Hypertension & $15(28 \%)$ \\
\hline Hyperlipidemia & $6.6(6.4,8.7)$ \\
\hline MELD & $139(137,141)$ \\
\hline Sodium (mEq/I), median (IQR) & $0.79(0.67,0.94)$ \\
\hline Creatinine (mg/dl), median (IQR) & $4.2(4,4.4)$ \\
\hline Albumin (g/dl), median (IQR) & $0.6(0.4,0.82)$ \\
\hline Total bilirubin (mg/dl), median (IQR) & $69(60,101)$ \\
\hline Alkaline phosphatase (U/I), median (IQR) & $26(20,36)$ \\
\hline AST (U/I), median (IQR) & $21(15,32)$ \\
\hline ALT (U/I), median (IQR) & $1(1,1.13)$ \\
\hline INR, median (IQR) & $207(158,261)$ \\
\hline Platelets (x 109 per I), median (IQR) & $44(83 \%)$ \\
\hline On any AIH treatment & $17(32 \%)$ \\
\hline Steroids & $32(60 \%)$ \\
\hline Azathioprine & $10(19 \%)$ \\
\hline Mycophenolate mofetil & $4(8 \%)$ \\
\hline Tacrolimus & \\
\hline Bul - body mass index, MEID & \\
\hline
\end{tabular}

BMI - body mass index, MELD - model for end-stage liver disease, AST - aspartate aminotransferase; ALT - alanine aminotransferase, INR - international normalized ratio, AlH - autoimmune hepatitis

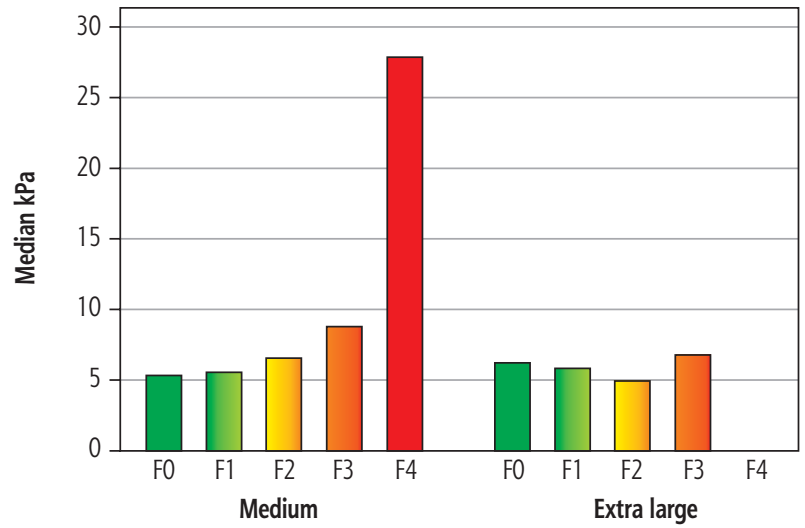

Fig. 1. Median $\mathrm{kPa}$ for fibrosis categories by probe size

Figure 1. Based on these data, the association between histologic fibrosis classification and $\mathrm{kPa}$ categories are presented in Table 2. When the M probe was used, we found a significant association between increasing $\mathrm{kPa}$ performed to evaluate the association between histologic fibrosis stage and $\mathrm{TE} \mathrm{kPa}$ categories for each probe type ( $\mathrm{M}$ or $\mathrm{XL}$ ) as well as pooled together. To assess for differences in BMI by probe type, we used the Wilcoxon rank-sum test.

Linear regression and correlation analysis were used to estimate projected associations between median $\mathrm{kPa}$ (as a continuous variable) and fibrosis categories when using the $\mathrm{M}$ probe. Univariate analysis of the variables in Table 1 was first performed to identify covariates potentially associated with median $\mathrm{kPa}$ scores for a given patient, using an $\alpha$ threshold $=0.10$ for selection for subsequent multivariable analysis. Because there appeared to be an exponential rather than linear relationship between median $\mathrm{kPa}$ and fibrosis category, median $\mathrm{kPa}$ was log transformed at this stage. We then performed multivariable regression analysis using a clinician-driven modeling approach, using a minimum Bayesian information criterion value to select a final model. This model included the fibrosis score and alkaline phosphatase level at the time of TE measurement as variables significantly associated with $\log ($ median $\mathrm{kPa})$. We then used this model to plot predicted median $\mathrm{kPa}$ values as a function of fibrosis score, with a $95 \%$ confidence band. Unless otherwise stated, an a level of 0.05 was used as the threshold for statistical significance in hypothesis testing. STATA/IC 15.1 (College Station, TX) was used for data management and analysis.

\section{Results}

\section{Patient characteristics}

A total of 60 patients with AIH were identified who received TE assessment and liver biopsy per the inclusion criteria. Of these, 2 had concomitant liver disease other than $\mathrm{AIH}$, and an additional 5 had unreliable TE measurements, yielding an analytic cohort of 53 patients per the selection criteria. The cohort was predominantly female with a median age of 56.3 years (IQR 44.4-63.5 years) and median MELD of 6.6 (IQR 6.4-8.7; Table 1). Median transaminase values at the time of TE were within the normal range (median AST 26, IQR 20-36; median ALT 21, IQR 15-32), but the majority of patients (83\%) were on active $\mathrm{AIH}$ treatment, with azathioprine being the most commonly prescribed medication (60\%).

\section{Association between transient elastography measurements and liver fibrosis}

Median $\mathrm{kPa}$ values as a function of histologic fibrosis score, stratified by probe type, are shown in 
Table 2. Fibrosis classification by probe type

\begin{tabular}{|c|c|c|c|c|c|c|c|}
\hline \multirow[t]{2}{*}{ Probe } & \multirow[t]{2}{*}{ Fibrosis } & \multicolumn{5}{|c|}{ kPa Category } & \multirow[b]{2}{*}{$p$-value } \\
\hline & & $\leqslant 5.2$ & 5.3-6.1 & $6.2-8.4$ & $8.5-14.6$ & $\geqslant 14.7$ & \\
\hline \multirow[t]{2}{*}{ Pooled } & Advanced & 3 & 2 & 3 & 5 & 5 & \multirow[t]{2}{*}{$0.02^{*}$} \\
\hline & Mild/mod & 10 & 10 & 7 & 5 & 3 & \\
\hline \multirow[t]{2}{*}{ Medium } & Advanced & 2 & 2 & 1 & 3 & 5 & \multirow[t]{2}{*}{$0.04 *$} \\
\hline & Mild/mod & 8 & 5 & 5 & 4 & 2 & \\
\hline \multirow[t]{2}{*}{ Extra large } & Advanced & 1 & 0 & 2 & 2 & 0 & \multirow[t]{2}{*}{0.40} \\
\hline & Mild/mod & 2 & 5 & 2 & 1 & 1 & \\
\hline
\end{tabular}

category and advanced fibrosis $(p=0.04)$. However, with use of the XL probe, there was no association between advanced fibrosis and increasing $\mathrm{kPa}$ category $(p=0.40)$. Median patient BMI, however, was significantly different between the $\mathrm{M}$ and XL probe groups (25.8, IQR 22.5-28.5 vs. 33.1, IQR 29.4-37.7; $p<0.001$ ). Among patients where the $\mathrm{M}$ probe was used, there was a strong correlation between $\mathrm{kPa}$ and fibrosis score $(\rho=0.42, p=0.01)$.

\section{Linear regression analysis}

In the final linear regression model, fibrosis score $(\beta=0.27, p=0.003)$ and alkaline phosphatase $(\beta=0.03$ per 10 -unit change, $p=0.028)$ were retained as variables significantly associated with $\log ($ median $\mathrm{kPa})$. The predicted association between fibrosis score and median $\mathrm{kPa}$ by TE, adjusted for alkaline phosphatase, is shown in Figure 2 and Table 3. Although median $\mathrm{kPa}$ increased with worsening fibrosis score, the increase was much more marked when fibrosis stage progressed from F3 to F4 (median $\mathrm{kPa} 10.1$ to 18.8 , respectively).

\section{Discussion}

This retrospective study demonstrates a significant association between $\mathrm{TE} \mathrm{kPa}$ values and histologic fibrosis staging on biopsy in a United States cohort, adding validity to the use of this non-invasive measure for assessing liver fibrosis in patients with AIH. Importantly, this significant association was only present when the M probe was used; there was no significant association with use of the XL probe. Significant differences in BMI between these two groups may explain these findings, which would be consistent with existing literature on TE inaccuracy in patients with increased BMI.

To the best of our knowledge, this study represents the first TE validation study in AIH patients conducted in a United States population. Prior cohort studies cor-

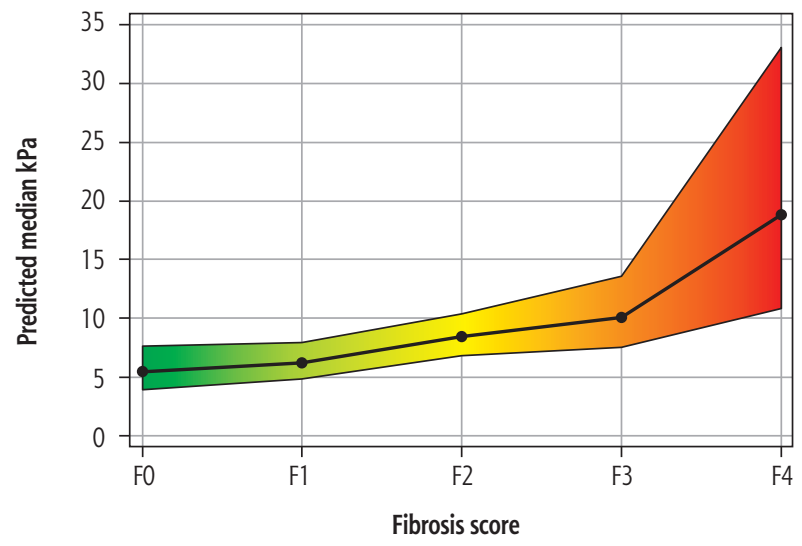

Fig. 2. Association between $\mathrm{kPa}$ and fibrosis stage with medium probe (with 95\% confidence band)

Table 3. Predicted median $\mathrm{kPa}$ values by fibrosis score using the medium probe, with $95 \%$ confidence intervals

\begin{tabular}{lcc}
\hline Fibrosis score & Median $\mathrm{kPa}$ & 95\% Confidence Interval \\
\hline F0 & 5.4 & $3.8-7.7$ \\
\hline F1 & 6.2 & $4.8-7.9$ \\
\hline F2 & 8.4 & $6.8-10.4$ \\
\hline F3 & 10.1 & $7.5-13.6$ \\
\hline F4 & 18.8 & $10.7-33.1$ \\
\hline
\end{tabular}

roborate these findings in Chinese and German populations. In a study of 30 patients by Wang et al., a significant positive correlation was found between degree of liver stiffness as assessed by TE and Scheuer fibrosis stage by biopsy. One patient had a failed attempt at TE measurement due to high BMI [10]. In a larger study of 108 patients by Guo et al., TE was found to be superior to other non-invasive markers, including the aspartate transaminase-to-platelet index (APRI) and a fibrosis index based on four factors (FIB-4), in detecting the stages of fibrosis in $\mathrm{AIH}$ patients previously determined through liver biopsy [12]. A study of 60 German patients with AIH demonstrated a strong positive correlation between TE measurements and 
histologic fibrosis stage ( $\rho=0.78, p<0.001)$ and identified an optimal $\mathrm{kPa}$ cutoff of 16 to identify patients with F4 fibrosis [13]. Finally, another Chinese study of 100 patients reported an optimal $\mathrm{kPa}$ of 12.5 in classifying F4 fibrosis [14]. Our results mirror those of these studies, as we found that a transition from F3 fibrosis to F4 fibrosis would be marked by approximate median $\mathrm{kPa}$ values of 10.1 to 18.8 , respectively. However, in contrast to the above work, our cohort included a mixed Caucasian and black population, which is more reflective of the United States AIH population [16], and explored the role of probe type and BMI in the analysis (discussed below).

The second major finding in our study was that TE measurements correlated strongly with fibrosis when using the $\mathrm{M}$ probe but were unreliable when using the XL probe. Based on existing literature, we believe that BMI differences between groups are likely to explain this observation, where reduced penetration of $\mathrm{TE}$ shear waves into the intrahepatic tissue reduces performance of the test [21]. Indeed, a prospective study of more than 10,000 patients indicated that liver stiffness measurements are unreliable in nearly one in five cases, often due to obesity [22]. Unreliable measurements ranged from $12 \%$ in patients with $\mathrm{BMI}<25$ to more than $50 \%$ in patients with $\mathrm{BMI} \geq 40$. This included patients with chronic hepatitis $\mathrm{B} / \mathrm{C}$, nonalcoholic fatty liver disease, alcoholic liver disease, and a miscellaneous category where etiology of liver disease was not specified. In our study, we observed poor performance beginning at a lower BMI range of approximately $>30$. This suggests that the impact of BMI may vary among different etiologies of liver disease, although this premise would need to be explored in future studies.

There are several limitations that we acknowledge in this study. First, this study includes a relatively small sample size and therefore significant differences may have been missed due to insufficient power. Second, there is a possibility of misclassification of exposure in our study. Although we restricted study inclusion to patients with AIH and no documented concomitant liver disease, it is possible that some patients carry additional undiagnosed chronic liver diseases such as alcoholic liver disease or non-alcoholic fatty liver disease, which could impact the results of this study. To address this, we performed detailed chart reviews and only identified two patients with concomitant liver disease, suggesting that this impact is likely minimal. Third, there is a possible misclassification of fibrosis by biopsy, as errors in staging may result from sampling or pathologist variation [5]. However, this limitation is shared with similar studies, would not be expected to bias the results in a systematic fashion, and the im- pact is somewhat reduced by pooling fibrosis groups. Fourth, the transaminase levels in our cohort were generally in the normal range (i.e. biochemical stability), indicating that there was minimal active hepatitis in this study. Indeed, the vast majority of patients were on maintenance therapy with azathioprine with long-established diagnoses of AIH. As such, the results may not generalize well to populations with high degrees of active inflammation (as might occur during an AIH flare), and we could not explore the impact of hepatic inflammation on TE measurement, which could theoretically impact the results [23]. However, this question has been addressed in prior literature [12], without a suggestion that hepatic inflammation substantially impacts TE readings in AIH patients. Furthermore, AST and ALT in our study were not retained as covariates significantly associated with median $\mathrm{kPa}$ measurements. Finally, as our study population primarily included middle-aged, female, Caucasian and black patients, the results may not be generalizable to all patients with $\mathrm{AIH}$.

In conclusion, our results support the use of TE as a reliable and non-invasive means of assessing liver fibrosis in patients with AIH. However, the reliability of TE may diminish in patients with increased BMI, even with use of the XL probe. Hence, caution should be exercised when assessing obese patients and further evaluation with biochemical and serological testing, imaging, and biopsy should be considered in the appropriate clinical context.

\section{Funding}

Nadim Mahmud is currently supported through a National Institutes of Health T32 grant (2-T32DK007740-21A1).

This study received Institutional Review Board approval from the University of Pennsylvania with a waiver of informed consent.

\section{Disclosure}

Authors report no conflict of interest.

\section{References}

1. Heneghan MA, Yeoman AD, Verma S, et al. Autoimmune hepatitis. Lancet 2013; 382: 1433-1444.

2. Hennes EM, Zeniya M, Czaja AJ, et al. Simplified criteria for the diagnosis of autoimmune hepatitis. Hepatology 2008; 48: $169-176$.

3. Gatselis NK, Zachou K, Koukoulis GK, Dalekos GN. Autoimmune hepatitis, one disease with many faces: etiopathogenetic, clinico-laboratory and histological characteristics. World J Gastroenterol 2015; 21: 60 . 
4. Afdhal NH, Bacon BR, Patel K, et al. Accuracy of fibroscan, compared with histology, in analysis of liver fibrosis in patients with hepatitis B or C: a United States multicenter study. Clin Gastroenterol Hepatol 2015; 13: 772-779.e773.

5. Bedossa P, Dargère D, Paradis V. Sampling variability of liver fibrosis in chronic hepatitis C. Hepatology 2003; 38: 1449-1457.

6. Tapper EB, Castera L, Afdhal NH. FibroScan (vibration-controlled transient elastography): where does it stand in the United States practice. Clin Gastroenterol Hepatol 2015; 13: 27-36.

7. Ganne-Carrié N, Ziol M, de Ledinghen V, et al. Accuracy of liver stiffness measurement for the diagnosis of cirrhosis in patients with chronic liver diseases. Hepatology 2006; 44: 1511-1517.

8. Nguyen-Khac E, Chatelain D, Tramier B, et al. Assessment of asymptomatic liver fibrosis in alcoholic patients using fibroscan: prospective comparison with seven non-invasive laboratory tests. Aliment Pharmacol Ther 2008; 28: 1188-1198.

9. Nahon P, Kettaneh A, Tengher-Barna I, et al. Assessment of liver fibrosis using transient elastography in patients with alcoholic liver disease. J Hepatol 2008; 49: 1062-1068.

10. Ziol M, Handra-Luca A, Kettaneh A, et al. Noninvasive assessment of liver fibrosis by measurement of stiffness in patients with chronic hepatitis C. Hepatology 2005; 41: 48-54.

11. Wang Q, Shen L, Qiu D, et al. Validation of transient elastography (Fibroscan) in assessment of hepatic fibrosis in autoimmune hepatitis. Zhonghua Gan Zang Bing Za Zhi 2011; 19: 782-784.

12. Guo L, Zheng L, Hu L, et al. Transient elastography (FibroScan) performs better than non-invasive markers in assessing liver fibrosis and cirrhosis in autoimmune hepatitis patients. Med Sci Monit 2017; 23: 5106.

13. Hartl J, Denzer U, Ehlken H, et al. Transient elastography in autoimmune hepatitis: Timing determines the impact of inflammation and fibrosis. J Hepatol 2016; 65: 769-775.

14. Xu Q, Sheng L, Bao H, et al. Evaluation of transient elastography in assessing liver fibrosis in patients with autoimmune hepatitis. J Gastroenterol Hepatol 2017; 32: 639-644.

15. Anastasiou OE, Büchter M, Baba HA, et al. Performance and utility of transient elastography and non-invasive markers of liver fiibrosis in patients with autoimmune hepatitis: A single centre experience. Hepatit Mon 2016; 16: e40737.

16. Manns MP, Czaja AJ, Gorham JD, et al. Diagnosis and management of autoimmune hepatitis. Hepatology 2010; 51: 2193 2213.

17. Boursier J, Zarski JP, de Ledinghen V, et al. Determination of reliability criteria for liver stiffness evaluation by transient elastography. Hepatology 2013; 57: 1182-1191.

18. Myers RP, Pomier-Layrargues G, Kirsch R, et al. Discordance in fibrosis staging between liver biopsy and transient elastography using the FibroScan XL probe. J Hepatol 2012; 56: 564-570.

19. Pang JX, Pradhan F, Zimmer S, et al. The feasibility and reliability of transient elastography using Fibroscan ${ }^{\oplus}$ : a practice audit of 2335 examinations. Can J Gastroenterol Hepatol 2014; 28: 143-149.

20. Ferraioli G, De Silvestri A, Reiberger T, et al. Adherence to quality criteria improves concordance between transient elastography and ElastPQ for liver stiffness assessment - A multicenter retrospective study. Dig Liver Dis 2018; 50: 1056-1061.

21. Weiss J, Rau M, Meertens J, et al. Feasibility of liver stiffness measurement in morbidly obese patients undergoing bariatric surgery using XL probe. Scand J Gastroenterol 2016; 51: 1263-1268.

22. Castéra L, Foucher J, Bernard PH, et al. Pitfalls of liver stiffness measurement: a 5-year prospective study of 13,369 examinations. Hepatology 2010; 51: 828-835.

23. Wong GL-H. Update of liver fibrosis and steatosis with transient elastography (Fibroscan). Gastroenterol Rep 2013; 1: 19-26. 